\title{
Odontologia como escolha: perfil de graduandos e perspectiva para o futuro profissional
}

Frederika Cartagena Machado*, Danielle Mariana de Almeida Souto**, Claudia Helena Soares Morais Freitas***, Franklin Delano Soares Forte****

\author{
* Cirurgiã-Dentista formadas pelo Curso de odontologia da \\ Universidade Federal da Paraíba \\ ** Cirurgiã-Dentista formadas pelo Curso de odontologia da \\ Universidade Federal da Paraíba \\ *** Professora Doutora do Departamento de Clínica e Odontologia \\ Social da Universidade Federal da Paraíba \\ **** Professor Doutor do Departamento de Clínica e Odontologia \\ Social da Universidade Federal da Paraíba
}

\section{RESUMO}

Objetivo: Verificar o perfil sócio-econômico dos acadêmicos do Curso de Odontologia da UFPB, destacando-se motivo de escolha profissional e percepção de mercado de trabalho. Participaram 235 acadêmicos, do primeiro ao décimo período. Material e Método: Realizou-se investigação quantitativa, baseada na aplicação de questionários estruturados. Os estudantes foram agrupados em dois: $\mathrm{G}_{1}\left(1^{\circ}, 2^{\circ}\right.$ e $3^{\circ}$ anos $)$ e $\mathrm{G}_{2}\left(4^{\circ}\right.$ e $5^{\circ}$ anos $)$. Resultados: A análise demonstrou que a maioria foi do sexo feminino $31,9 \% \mathrm{G}_{1}$ e $22 \mathrm{G}_{2}$. A maioria em ambos os grupos concluiu o ensino médio em escola privada. Em relação a renda 58,3\% $\mathrm{G}_{1} 51,5 \% \mathrm{G}_{2}$ e são provenientes de famílias com renda acima de seis salários mínimo. A maioria não trabalhava, tinha computador em casa e residiam com suas famílias. A formação profissional voltada para o trabalho foi citada por $52,3 \% \mathrm{G}_{1}$ e $73,8 \% \mathrm{G}_{2}$. Dentre os motivos de escolha da profissão mais citados esteve a realização profissional e pessoal com $39,1 \%$ para o $G_{1}$ e $27,9 \%$ para o $G_{2}$. Não havendo diferenças entre os grupos $(p=0,175)$. A maioria pretende trabalhar no setor público e privado independente do grupo. Relataram a saturação de profissionais no mercado de trabalho $(p=0,036)$, a condição financeira da população $(p=0,043)$ e a falta de informação $(p=0,419)$ como possíveis dificuldades no exercício da profissão. Conclusões: Os acadêmicos são provenientes de famílias com boas condições sócio-econômicas; realizaram escolha profissional baseados na vocação; relataram intenção de realizar capacitações; expressaram preocupação com o mercado de trabalho.

\section{DESCRITORES}

Recursos humanos em odontologia. Mercado de trabalho. Formação profissional.

$\mathbf{N}$ os últimos anos os profissionais de saúde bucal foram inseridos nos diversos níveis de atenção a saúde e na gestão de serviços de saúde no Brasil, o que reforça a necessidade de reflexão sobre a prática profissional com responsabilidade e ética na produção do cuidado. Sendo assim, é importante saber quais as expectativas dos dentistas em relação a profissão assim como, quais os motivos de se fazer odontologia, assim como compreender os esforços da profissão para responder as demandas da sociedade.

O Brasil teve um grande crescimento no número de cursos de odontologia, em 1996 tinha 90 cursos, aumentando para 191 em 2009, sendo a maioria localizado na região Sudeste (São Paulo, Rio de Janeiro e Minas Gerais). Se por um lado existe a oferta na demanda na formação de profissionais, a pesquisa nacional por amostra de domicílios publicada em 2010 revelou que $11,6 \%$ dos brasileiros não receberam tratamento odontológico e $98 \%$ dos participantes da pesquisa com rendimento de mais de cinco salários mínimos já foi ao consultório do dentista, por outro lado, dentre os que ganham até um quarto de salário mínimo esse percentual foi de $73 \% .^{1}$ Quando considerado apenas as consultas feitas no último ano 
existe uma diferença expressiva entre o grupo de maior renda $67,2 \%$ com a de menor $28,5 \%$. $^{1}$

Neste contexto, existe ainda a crença de que o número maior de profissionais seria a solução para resolução dos problemas de saúde bucal das pessoas. Em países desenvolvidos e em desenvolvimento a quantidade de profissionais não é tão importante assim na determinação de melhores condições do padrão de saúde bucal dos indivíduos. ${ }^{2}$

Além dessa questão existe a concentração de profissionais nos grandes centros urbanos. ${ }^{3,4}$ No Brasil existem registrados no Conselho Federal de Odontologia 219.575 dentistas. Do total, 2.859 tem seu registro no Conselho Regional da Paraíba, sendo 2.035 de inscrição principal, com $58,5 \%$ dos profissionais concentrados na capital. ${ }^{4}$ Atualmente, existem três Universidades Públicas que formam os profissionais no estado da Paraíba, com campus localizados em João Pessoa, Campina Grande, e quatro Instituições (duas publicas e duas privadas) que ofertaram cursos de odontologia recentemente que não possui turmas formadas.

Em 2002 foi implantado na Universidade Federal da Paraíba (UFPB) o novo Projeto Pedagógico do Curso de Odontologia e entre seus objetivos está a formação de recursos humanos dentro de padrões éticos e humanos, voltados para o perfil epidemiológico da população como também para o Sistema de Saúde vigente no Brasil.

Diante deste contexto, o objetivo desse trabalho foi analisar o perfil sócio-econômico-cultural do acadêmico do Curso de Odontologia da Universidade Federal da Paraíba, buscando identificar o motivo de sua escolha pela profissão e perspectivas para o futuro profissional.

\section{MATERIAL E MÉTODOS}

Este é um estudo descritivo, transversal com abordagem quantitativa. Os sujeitos da pesquisa foram acadêmicos, de ambos os gêneros, do Curso de Odontologia da Universidade Federal da Paraíba, regularmente

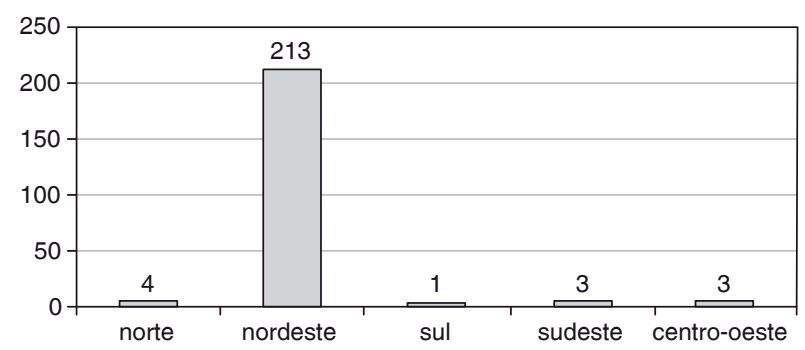

Gráfico 1 - Distribuição dos estudantes segundo região de origem. João Pessoa, 2008. matriculados no primeiro semestre do ano de 2007. A amostra foi calculada considerando-se grau de confiança de $95 \%$, poder do teste de $50 \%$ e erro aceitável de $5 \%$. Foi acrescido $20 \%$ para compensar possíveis perdas amostrais. Participaram da amostra 235 acadêmicos do curso de odontologia da UFPB. O processo de amostragem desse estudo foi probabilística casual simples sem reposição. Como o curso é semestral a amostra foi constituída por semestre, posteriormente agrupada por ano e então dividida em dois grupos:

- $\mathrm{G}_{1}$ : aqueles matriculados no primeiro e segundo ano do curso;

- $\mathrm{G}_{2}$ : os que estavam matriculados no terceiro, quarto e quinto ano.

A divisão se deu dessa forma, em função do inicio das clinicas odontológicas ser um fator importante na formação profissional, podendo influenciar suas decisões e percepções ao responder ao questionário. Para tanto foi solicitado a Coordenação do Curso uma lista com os nomes dos alunos.

Inicialmente foram explicados os objetivos do estudo e depois aplicado o instrumento de coleta de dados. O questionário foi adaptado de Freire et al. $(1995)^{5} \mathrm{e}$ Freitas e Nakayama $(1995)^{6}$ com perguntas objetivas e subjetivas. Este questionário foi previamente aplicado a seis estudantes e a um docente do Curso de Odontologia da UFPB a título de pré-teste. Após a análise, consideraram o questionário de fácil compreensão, sugeriramalgumasmodificaçõesenãoapontaramproblemas em respondê-lo. Dessa forma, incluímos e re-estruturamos algumas questões, chegando-se à versão final.

$\mathrm{O}$ instrumento de coleta abordou questões que vão desde a caracterização sócio-econômica, motivos para escolha da profissão e expectativas para o futuro profissional. Os dados foram analisados pela estatística descritiva e submetidos ao teste estatístico quiquadrado considerando significativo ao nível de $5 \%$.

A proposta do estudo baseou-se no cumprimento dos princípios éticos contidos na Declaração de Helsink (2000), além do atendimento da resolução 196/1996 do Conselho Nacional de Saúde. O estudo foi aprovado pelo Comitê de ética em Pesquisa do Centro de Ciências da Saúde da Universidade Federal da Paraíba com o numero 440/06. Todos os participantes da pesquisa assinaram o Termo de Consentimento Livre e Esclarecido.

\section{RESULTADOS}

Em ambos os grupos a maioria dos entrevistados $51,5 \% \mathrm{G}_{1}$ e $34,0 \% \mathrm{G}_{2}$ concluíram o ensino médio em 
unidade escolar privada. Além disso, 41,5\% $\mathrm{G}_{1} \mathrm{e}$ $28,9 \%$ são provenientes de famílias com renda acima de 6 salários mínimos. A maioria das mães e dos pais tem curso superior (Tabela 1 ).

Do total de alunos pesquisados observa-se que a maioria reside com sua família na cidade de João Pessoa. A maioria $35,5 \% \mathrm{G}_{1}$ e $18,3 \% \mathrm{G}_{2}$ usa o transporte coletivo da cidade para se locomover até a Universidade. Cerca de $48,9 \% \mathrm{G}_{1}$ e $33,6 \% \mathrm{G}_{2}(\mathrm{p}=0,116)$ tem computador em casa e o utiliza em sua maioria para entretenimento, trabalhos escolares e profissional. A maioria $59,1 \% \mathrm{G}_{1}$ e $36,9 \% \mathrm{G}_{2}$ apenas estuda e não exerce atividade profissional remunerada (Tabela 2).

Quando se refere as expectativas em relação ao curso universitário, os estudantes destacaram a formação profissional voltada para o trabalho, formação teórica voltada para a pesquisa e conhecimento para compreender melhor o mundo. Não foram observadas diferenças entre os grupos estudados $(\mathrm{p}<0,05)$. Os motivos que levaram os estudantes a escolher a Odontologia como profissão podem ser observados na Tabela 3, predominando a realização pessoal e profissional, não sendo verificada diferença estatisti- camente significante. As principais dificuldades relatadas para o exercício da profissão foram:

- saturação do mercado de trabalho,

- falta de informação da população e

- condição financeira da população.

Observamos que a expectativa dos estudantes para a inserção no mercado de trabalho é em sua maioria $48,3 \% \mathrm{G}_{1}$ e $\% 28,4 \% \mathrm{G}_{2}$ no consultório particular associado ao público. Os estudantes pretendem após o término do curso, em sua maioria realizar cursos de pós-graduação lato sensu (aperfeiçoamento e especialização) (Tabela 4).

\section{DIsCusSÃo}

A presença de muitos estudantes paraibanos e também de outros estados nordestinos representa a inserção da instituição de ensino superior na comunidade na qual está inserida. Evidencia-se dessa forma, a importância e a responsabilidade institucional na formação e capacitação de recursos humanos em saúde. Alguns fatores como capacitação, formação e compromisso influenciam na qualidade da prestação

Tabela 1 - Perfil sócio econômico dos estudantes de Odontologia da UFPB. João Pessoa, 2008.

\begin{tabular}{|c|c|c|c|c|c|c|}
\hline & \multirow{2}{*}{ Perfil } & \multicolumn{2}{|c|}{ Grupo 1} & \multicolumn{2}{|c|}{ Grupo 2} & \multirow[t]{2}{*}{$\mathbf{p}$} \\
\hline & & $\mathbf{n}$ & $\%$ & $\mathbf{n}$ & $\%$ & \\
\hline \multirow{2}{*}{ Sexo } & Masculino & 67 & 28,9 & 40 & 17,2 & 0,346 \\
\hline & Feminino & 74 & 31,9 & 51 & 22,0 & \\
\hline \multirow{3}{*}{ Estado civil } & Solteiro & 134 & 58,3 & 83 & 36,1 & \\
\hline & Casado & 3 & 1,3 & 6 & 2,6 & - \\
\hline & Outros & 4 & 1,7 & - & - & \\
\hline \multirow{3}{*}{ Ensino médio } & Todo em escola pública & 12 & 5,1 & 4 & 1,7 & \\
\hline & Todo em escola privada & 121 & 51,5 & 80 & 34,0 & - \\
\hline & Escola privada e pública & 11 & 4,6 & 7 & 3,0 & \\
\hline \multirow{4}{*}{ Renda familiar } & Até 1 Salário mínimo & 3 & 1,3 & 1 & 0,4 & \\
\hline & De 1 a 3 salários mínimos & 34 & 14,5 & 19 & 8,1 & - \\
\hline & De 3 a 6 salários mínimos & 9 & 3,8 & 1 & 0,4 & \\
\hline & Acima de 6 salários mínimos & 97 & 41,5 & 70 & 29,9 & \\
\hline \multirow{2}{*}{$\begin{array}{l}\text { Escolaridade } \\
\text { materna }\end{array}$} & Até 8 anos & 11 & 4,7 & 1 & 0,4 & 0,022 \\
\hline & Acima de 8 anos & 132 & 56,7 & 89 & 38,2 & \\
\hline \multirow{2}{*}{$\begin{array}{l}\text { Escolaridade } \\
\text { paterna }\end{array}$} & Até 8 anos & 17 & 7,4 & 5 & 2,2 & 0,082 \\
\hline & Acima de 8 anos & 125 & 54,1 & 84 & 36,4 & \\
\hline \multirow{4}{*}{$\begin{array}{c}\text { Transporte para a } \\
\text { universidade }\end{array}$} & Carro ou motocicleta próprios & 21 & 8,9 & 15 & 6,4 & \\
\hline & Carro dos pais & 21 & 8,9 & 22 & 9,4 & - \\
\hline & Carona com amigos e vizinhos & 9 & 3,8 & 6 & 2,6 & \\
\hline & Transporte coletivo (ônibus) & 84 & 35,7 & 43 & 18,3 & \\
\hline
\end{tabular}


Tabela 2 - Residência e uso de computador por estudantes de odontologia da UFPB. João Pessoa, 2008.

\begin{tabular}{|c|c|c|c|c|c|}
\hline \multirow{2}{*}{ Residência } & \multicolumn{2}{|c|}{ Grupo 1} & \multicolumn{2}{|c|}{ Grupo 2} & \multirow{2}{*}{$\mathbf{p}$} \\
\hline & $\mathbf{n}$ & $\%$ & $\mathbf{N}$ & $\%$ & \\
\hline Com família (pais, irmãos) & 93 & 39,7 & 59 & 25,2 & \\
\hline Com família (cônjuge) & 3 & 1,3 & 3 & 1,3 & \\
\hline Com parentes (primos, tios, padrinhos) & 18 & 7,7 & 8 & 3,4 & - \\
\hline Com amigos ou colegas & 19 & 8,1 & 14 & 6,0 & \\
\hline Pensionato ou quitinete & 7 & 3,0 & 4 & 1,7 & \\
\hline Alojamento universitário & 3 & 1,3 & 2 & 0,9 & \\
\hline \multicolumn{6}{|l|}{ Tem computador em casa? } \\
\hline Sim & 115 & 48,9 & 79 & 33,6 & 0,116 \\
\hline Não & 29 & 12,3 & 12 & 5,1 & \\
\hline \multicolumn{6}{|l|}{ Para quê utiliza o computador?* } \\
\hline Não utilizo computador & 4 & 1,7 & 1 & 0,4 & \\
\hline Trabalhos escolares & 11 & 4,7 & 3 & 1,3 & - \\
\hline Trabalhos profissionais & 1 & 0,4 & 0 & 0 & \\
\hline Entretenimento e trabalhos & 125 & 53,6 & 86 & 36,9 & \\
\hline \multicolumn{6}{|l|}{ O estudante trabalha? } \\
\hline Sim (até 30 horas semanais) & 4 & 1,7 & 3 & 1,3 & \\
\hline Sim (mais de 30 horas semanais) & 1 & 4 & 0 & 0 & - \\
\hline Não & 139 & 59,1 & 88 & 37,4 & \\
\hline
\end{tabular}

*opção de mais de um registro.

Tabela 3 - Motivos de escolha profissional pelos estudantes de

Odontologia da UFPB. João Pessoa, 2008.

\begin{tabular}{|c|c|c|c|c|c|}
\hline & \multicolumn{2}{|c|}{ Grupo 1} & \multicolumn{2}{|c|}{ Grupo 2} & \multirow{2}{*}{$\mathbf{p}$} \\
\hline Qual sua expectativa em relação ao curso universitário?* & $\mathbf{n}$ & $\%$ & $\mathbf{n}$ & $\%$ & \\
\hline Formação profissional voltada para o trabalho & 123 & 52,3 & 7 & 73,8 & 0,867 \\
\hline Aquisição de cultura geral ampla & 8 & 3,4 & 9 & 3,8 & 0,211 \\
\hline Formação acadêmica para melhorar atividade prática atual & 7 & 3,0 & 10 & 4,3 & 0,77 \\
\hline Conhecimento para melhorar o grau de instrução & 5 & 2,1 & 10 & 4,3 & 0,022 \\
\hline Formação teórica voltada para a pesquisa & 10 & 4,3 & 13 & 5,5 & 0,065 \\
\hline Conhecimento para compreender melhor o mundo & 10 & 4,3 & 13 & 5,5 & 0,065 \\
\hline Possibilidade de obter melhores salários & 0 & 0 & 4 & 1,7 & - \\
\hline \multicolumn{6}{|l|}{ Qual foi o motivo de escolha para o curso de odontologia?* } \\
\hline Realização pessoal e profissional & 91 & 39,1 & 65 & 27,9 & 0,175 \\
\hline $\begin{array}{l}\text { Segurança e tranqüilidade no futuro, posição social e } \\
\text { conforto financeiro }\end{array}$ & 28 & 12,0 & 13 & 5,6 & 0,316 \\
\hline Influência de CD parentes ou amigos & 15 & 6,4 & 10 & 4,3 & 0,881 \\
\hline Grande mercado de trabalho & 14 & 6,0 & 6 & 2,6 & 0,407 \\
\hline \multicolumn{6}{|c|}{ Qual (is) dificuldade(s) você imagina encontrar no exercício profissional?* } \\
\hline Falta de informação da comunidade & 47 & 20,3 & 32 & 13,8 & 0,043 \\
\hline Condição financeira da população & 37 & 15,8 & 25 & 10,7 & 0,419 \\
\hline Falta de preparo do dentista & 11 & 4,7 & 11 & 4,7 & 0,181 \\
\hline Acho que não terei problemas & 4 & 1,7 & 2 & 0,9 & 0,571 \\
\hline Saturação do mercado de trabalho & 88 & 37,6 & 67 & 28,6 & 0,036 \\
\hline
\end{tabular}

*opção de mais de um registro. 
Odontologia como escolha: perfil de graduandos e perspectiva para o futuro profissional • Machado FC, Souto DMA, Freitas CHSM, Forte FDS

Tabela 4

Expectativas para o futuro profissional e investimento em capacitação. João Pessoa, 2008.

\begin{tabular}{|c|c|c|c|c|}
\hline & \multicolumn{2}{|c|}{ Grupo 1} & \multicolumn{2}{|c|}{ Grupo 2} \\
\hline Ao término do curso você pretende trabalhar?* & $\mathbf{n}$ & $\%$ & $\mathbf{n}$ & $\%$ \\
\hline Consultório particular alugado com convênios & 2 & 0,9 & 1 & 0,4 \\
\hline Consultório particular alugado sem convênios & 3 & 1,3 & 0 & 0 \\
\hline Consultório particular próprio sem convênios & 6 & 2,6 & 1 & 0,4 \\
\hline Consultório particular próprio com convênios & 9 & 3,9 & 2 & 0,9 \\
\hline Consultório particular e serviço público & 112 & 48,3 & 66 & 28,4 \\
\hline Serviço público & 17 & 7,3 & 22 & 9,5 \\
\hline \multicolumn{5}{|c|}{ Quanto aos estudos, após a conclusão deste curso, o que pretende?* } \\
\hline Não fazer nenhum outro curso & 2 & 0,9 & 0 & 0 \\
\hline Fazer outro curso de graduação & 6 & 2,6 & 14 & 6,0 \\
\hline Fazer cursos de aperfeiçoamento e especialização & 98 & 42,3 & 64 & 27,6 \\
\hline Fazer curso de mestrado e doutorado na mesma área & 56 & 24,1 & 46 & 20,0 \\
\hline Fazer curso de mestrado e doutorado em outra área & 2 & 0,9 & 2 & 0,9 \\
\hline
\end{tabular}

*opção de mais de um registro.

de serviços em saúde.

Observou-se na presente pesquisa a forte presença do sexo feminino, independente dos $G_{1}$ e $G_{2}$. A força do trabalho das mulheres também foi observada por outros estudos. ${ }^{4-14}$ Isso indica uma mudança não só na odontologia, mas uma tendência em todas as áreas, a força de trabalho feminino na economia e na produção. Dados nacionais publicados em 2010 demonstraram a feminização do trabalho em quase todos os estados da federação, com exceção para Acre e Santa Catarina. Na Paraíba 67\% dos profissionais são mulheres. ${ }^{4}$

Na maioria dos estados brasileiros os profissionais cirurgiões-dentistas são jovens de 26 a 35 anos, ${ }^{4}$ com concentração na região Sul e Sudeste, especialmente no estado de São Paulo, atuando como clínicos gerais e/ou especialistas nos seus consultórios. ${ }^{4,7,8}$ A Pesquisa do Instituto Brasileiro de Estudos e Pesquisas Socioeconômicas (INBRAPE) ${ }^{9}$ verificou que $57 \%$ dos cirurgiões-dentistas do Brasil são mulheres. Na presente pesquisa, quanto ao estado civil observou-se que a maioria era solteira, refletindo uma tendência da população brasileira em casar-se mais tarde priorizando a construção de uma formação profissional e inserção no mercado de trabalho.

Independente de ser do $G_{1}$ ou $G_{2}$, os indicadores socioeconômicos dos estudantes e suas famílias revelaram boas condições. Dessa forma, pode-se observar que o nível sócioeconômico dos acadêmicos pode ser considerado bastante privilegiado se considerada a escolaridade materna e paterna e a renda familiar mensal, a posse de computador e o transporte para a universidade. ${ }^{16}$ Essa condição pode trazer mais con- forto ao estudante no sentido de desenvolver suas atividades no curso de forma de dedicação exclusiva, na aquisição de livros, e equipamentos necessários no desempenho de suas atividades, também pode significar facilidade de deslocamento para os cenários diversos de práticas nas atividades intra e extramuros. Discussões precisam ser feitas no sentido de ampliar o acesso ao curso a classes com menores indicadores socioeconômicos.

Essa questão tem movimentado nos últimos anos diversos instituições brasileiras inclusive a UFPB, não só relacionado à odontologia, mas o acesso a cursos de nível superior. A partir de 2011, o processo seletivo da UFPB tem a modalidade de ingresso por reserva de vagas. Sendo assim, a presente pesquisa subsidiará novas comparações futuras, assim como discussões dessa modalidade de ingresso por reserva de vagas. Possivelmente o perfil socioeconômico do estudante irá mudar especialmente oriundos de escolas públicas do ensino médio.

Os estudantes relataram sua opção pelo curso de odontologia por vocação e por acreditarem ser uma profissão compensadora em termos financeiros. Outros estudos nacionais também apontaram essa perspectiva., ${ }^{71,16-18}$ Outros fatores têm sido citados pelos acadêmicos como no Ceará, onde se observou a escolha de curso por aptidão $(37 \%)$ e $32,9 \%$ por estar relacionado à saúde. ${ }^{19}$ Em outra pesquisa realizada em Lages - $\mathrm{SC}^{16}$ os alunos destacaram como motivos da escolha pela odontologia a realização profissional e pessoal $(45,4 \%)$ e $18,8 \%$ pelo interesse em atuar na comunidade. A sobrevivência econômica foi o fator mais destacado no estudo em Goiás. ${ }^{5}$ Em Minas Ge- 
rais ${ }^{20}$ os estudantes relataram que a autonomia foi o motivo mais citado para a escolha profissional, outros destaques foram curso da área biológica e pelo retorno financeiro. Essa perspectiva de ser uma profissão liberal, boa renda e independência foram aspectos destacados por profissionais em recente pesquisa em Pernambuco, João Pessoa - PB e Peru. ${ }^{18-20-21}$

A intenção de inserção no mercado de trabalho dos estudantes é no setor publico e privado. Apesar de jovens, os acadêmicos expressam a preocupação com dificuldades no mercado de trabalho em odontologia ao relatarem a necessidade de inserção no setor público e privado suplementar, estudos em diversas partes do país apontam a mesma demanda. ${ }^{5,18}$ Em outro estudo realizado em João Pessoa - PB ${ }^{(24)}$ verificou-se que $78,4 \%$ dos cirurgiões-dentistas trabalham em consultório, $59 \%$ têm emprego público e 9,9\% emprego privado. Dos entrevistados $61,6 \%$ admitiram trabalhar com convênios. No estudo do SB Brasil ${ }^{23}$ o setor público foi o maior prestador de serviços odontológicos $55,6 \%, 48,1 \%$ e $40,5 \%$ nas faixas etárias 15-19, 35-44 e 65-74 anos respectivamente. Estudo em Fortaleza - $\mathrm{CE}^{8}$ com egressos observou que a maioria se inseriu no setor público, sem vinculo empregatício e como prestador de serviço. A inserção no mercado de trabalho se deu logo após a formatura.

Nos últimos anos, percebem-se, no exercício da profissão, diversas mudanças nos cenários de atuação como:

- implantação e ampliação de serviços públicos odontológicos nas diversas esferas do governo e na rede de atenção compreendidos pelo SUS e da política nacional de saúde bucal do Governo Federal,

- criação de serviços especiais odontológicos em empresas, associações e sindicatos,

- expansão dos planos odontológicos, o que de certa forma compromete a atuação do serviço privado, ${ }^{7,11,18}$

- aumento da oferta de especializações e consequentemente tecnologia disponível no mercado.

Todos esses movimentos são percebidos pelos estudantes durante a trajetória no curso, os quais influenciam a percepção sobre o mercado de trabalho futuro.

O PPC do curso de odontologia oferta como cenário de aprendizagem os estágios supervisionados no SUS, em seus diversos níveis de atenção. O objetivo é desenvolver competência e habilidades para o trabalho em equipe, procurando desenvolver as atividades clínicas individuais e as ações com grupos operativos nos equipamentos sociais adscritos as unidades de saúde da família, buscando a integralidade da atenção em saúde bucal.

Dentre as dificuldades relatadas para o futuro exercício profissional destacaram-se a saturação do mercado de trabalho, condição financeira da população e falta de informação da comunidade. Segundo dados recentemente publicados, ${ }^{4}$ a proporção dentista/população em João Pessoa é de 1/378 e concentração de $58,5 \%$ dos trabalhadores na capital. A fixação de profissionais de saúde no interior, especialmente da equipe mínima da atenção primária é uma demanda para discussões. A implantação de políticas nessa perspectivas é importante, no sentindo de melhorias na qualidade de trabalho dos profissionais, implantação de serviços, consequentemente ampliação de postos de trabalho, melhoria nas condições salariais, investimento de recursos humanos em capacitações e educação permanente em saúde, oferta de pós-graduação na forma de especialização e residência multiprofissional em saúde e hospitalar de forma descentralizada dos grandes centros urbanos são eixos importantes para discussão de gestores, trabalhadores da saúde, instituição de ensino superior ou centro formadores, associações e conselho representativo da classe odontológica e comunidade em geral.

Assim, esse cenário do mercado de trabalho e as formas de inserção profissional determinam alterações nas relações de trabalho e sua clientela. Neste contexto, há formas distintas de captação, redefinição do tipo, fluxo e acesso dos sujeitos aos profissionais. ${ }^{11,18}$ Isso é percebido não só pela população em geral, mas também pelos futuros profissionais. Nesta perspectiva existe um esforço e um direcionamento de resoluções do conselho nacional de ensino na mudança na formação dos profissionais de Saúde bucal, de acordo com o sistema de saúde público vigente no país (SUS).

A aspiração salarial após cinco anos de trabalho, neste estudo, foi semelhante a estudos desenvolvidos nacionalmente, ${ }^{11,16,25}$ ou seja, receber entre 6 a 10 salários mínimos. Na pesquisa do CFO de 2003, mais de $48 \%$ dos Dentistas relataram renda familiar mensal 3.600,00; destacando-se a prática privada com convênios/credenciamentos, o que poderiam ser percebidos como forma de assalariamento indireto. ${ }^{9}$ Recente pesquisa ${ }^{4}$ analisou que na região norte $19 \%$ dos dentistas declarou maior renda anual (mais que 72 mil/ano), na Paraíba esse valor é de apenas $6 \%$ dos 
profissionais. A relação anual de informações sociais do ministério do trabalho e emprego demonstrou que a hora de trabalho no nordeste é de $\mathrm{R} \$ 57,47$.

A maioria dos estudantes tem intenção de realizar cursos de pós-graduação mais na perspectiva de cursos latu sensu (aperfeiçoamento e especialização). É interessante pontuar esse aspecto, pois, mesmo sem exercer a atividade como profissionais já relatam a demanda de cursos de pós-graduação, fato também observado por outros estudos nacionais. ${ }^{5,6,11,16}$ É importante que se investigue essa demanda, por exemplo, se já se percebe na graduação lacunas de conhecimentos e habilidades, que serão barreiras para o exercício da profissão e como enfrentar essa demanda no curriculo.

Associado a esses fatores é importante destacar ainda a insegurança sentida por alguns recém formados no enfrentamento do mercado de trabalho e as deficiências existentes nos cursos de graduação em termos de alcançar as habilidades, competências e conhecimentos orientados pelas diretrizes nacionais e para as demandas da comunidade. Muitos cursos têm implementadas novas propostas pedagógicas e essas mudanças têm sido percebidas, embora com diferentes níveis de avanços. Por outro lado, em odontologia assim como em outras profissões existe a constante necessidade de atualização e aperfeiçoamento, em função das mudanças operadas na estruturação do sistema de saúde, dos avanços tecnológicos e das mudanças de paradigmas da sociedade.

Nesta perspectiva esse estudo foi importante ferramenta de diagnóstico na definição do perfil acadêmico do curso de odontologia da UFPB, norteador para discussão desse egresso no mercado de trabalho na região. Especialmente porque o cirurgião-dentista pode atuar em diversos campos, sendo importante a reflexão sobre a formação de profissionais de saúde e sua articulação com educação permanente, equipe multiprofissional, desenvolvimento de ações intersetoriais e o trabalho na gestão de serviços.

\section{CONCLUSÕES}

O estudo evidenciou que a maioria dos acadêmicos do curso de odontologia eram adultos jovens, do sexo feminino, solteiros, e provenientes de famílias com boas condições sócioeconômica. A expansão do mercado de trabalho público e a oportunidade de melhor remuneração, inserção no mercado de trabalho em associação privado e público são fatores destacados pelo conjunto amostral. A vocação foi um dos fatores mais citados pelos estudantes para a escolha profissional. A intenção da realização de capacitações latu e stricto-sensu foi expressa pela maioria. Os acadêmicos percebem e relatam preocupação em relação a perspectiva de mercado de trabalho futuro sendo essa demanda coerente com a realidade brasileira.

\section{ABSTRACT}

Dentistry as a choice: profile of undergraduate students and prospects for the future professional

Objective: The aim of this study was to assess the socioeconomic profile of students from the Dental School of the Federal University of Paraíba, highlighting why they chose their profession and how they perceive the job market. A total of 235 students participated in the study, from the first to the tenth terms. Material and Methods: Quantitative research was conducted, based on structured questionnaires. Students were grouped into two groups: $\mathrm{G}_{1}\left(1^{\text {st }}\right.$ and $2^{\text {nd }}$ years $)$ and $\mathrm{G}_{2}\left(3^{\text {rd }}, 4^{\text {th }}\right.$ and $5^{\text {th }}$ years $)$. Results: The analysis showed that most of the students were female: $31.9 \%$ of $\mathrm{G}_{1}$ and $22 \%$ of $\mathrm{G}_{2}$. Most of the students from both groups finished high school in a private school. Regarding income, $58.3 \%$ of $\mathrm{G}_{1}$ and $51.5 \%$ of $\mathrm{G}_{2}$ were from families with incomes over six minimum wages. Most of the students did not work, used a computer at home and lived with their families. Professional training for work purposes was cited by $52.3 \%$ of $\mathrm{G}_{1}$ and $73.8 \%$ of $\mathrm{G}_{2}$. Among the most frequently cited reasons for choosing the profession were professional and personal achievement, with $39.1 \%$ for $\mathrm{G}_{1}$ and $27.9 \%$ for $G_{2}$. There was no differences between the groups $(p=0.175)$. Most of the students want to work in the public and the private sectors, regardless of the group. Both groups reported the saturation of the labor market $(p=0.036)$, the financial situation of the population $(p=0.043)$ and lack of information $(p=0.419)$ as potential difficulties in exercising their profession. Conclusions: The students were from families of a good socioeconomic status, made career choices based on their calling, reported their intention to perform training, and expressed concern regarding the labor market.

\section{DESCRIPTORS}

Professional practice. Labor market. Professional training.

\section{REFERÊNCIAS}

1. Pesquisa nacional por amostra de domicílios. Instituto Brasileiro de Geografia e Estatística. Um panorama da saúde no Brasil: acesso e utilização dos serviços, condições de saúde e fatores de risco e proteção à saúde 2008 Acesso e utilização de 
Odontologia como escolha: perfil de graduandos e perspectiva para o futuro profissional • Machado FC, Souto DMA, Freitas CHSM, Forte FDS

serviços de saúde: 2010. 245p.

2. Nadanovsky P, Sheiham A. The relative contribution of dental services to the changes and geographical variations in caries status of 5- and 12-year-old children in England and Wales in the 1980s. Community Dental Health 1994;11: 215-223.

3. Instituto Brasileiro de Geografia e Estatística. Censo demográfico 2000: primeiros resultados da amostra. http://www.ibge. gov.br (acessado em 20/11/08).

4. Morita MC, Haddad A E, Araujo ME. Perfil atual e tendências do Cirurgião-Dentista Brasileiro. Maringá: dental Press. 2010. $96 \mathrm{p}$.

5. Freire MCM, Souza CS, Pereira HR. O perfil do acadêmico de Odontologia da Universidade Federal de Goiás. Divulgação em Saúde para Debate 1995;10:15-20.

6. Freitas SFT, Nakayama MH. Um perfil do estudante de Odontologia no estado de São Paulo. Divulgação em Saúde para Debate 1995;10:29-37.

7. Moysés SJ. Políticas de saúde e formação de recursos humanos em odontologia. Revista da ABENO 2004; 4(1): 30-37.

8. Pinheiro VC, Menezes LMB, Aguiar ASW, Moura WVB, Almeida MEL, Pinheiro FMC. Inserção dos egressos do curso de odontologia no mercado de trabalho. Revista gaucha de odontologia 2011 59(2): 277-283.

9. Conselho Federal de Odontologia. Instituto Brasileiro de Pesquisas sócio-econômicas. Perfil do Cirurgião-Dentista no Brasil. 2003.

10. Carvalho DR. Motivações e expectativas para o curso e para o exercício da odontologia. Rev. Assoc. Paul. Cir. Dent. 1997; 51 (4): 345-9.

11. Costa ICC, Marcelino G, Saliba NA. Perspectivas de um grupo de alunos de odontologia sobre a profissão no terceiro milênio. Rev ABOPREV 1999; 2(1):38-45.

12. Junqueira JC, Colombo CED, Tavares PG, Rocha RF, Carvalho YR, Rodrigues JR. Quem é e o que pensa o graduando de odontologia. Rev Odontol UNESP 2002;31(2):269-84.

13. Nicodemo D, Naressi WG. O perfil do aluno de odontologia - do ingresso à sua graduação. Rev Odonto Ciência 2002; $17(36): 135-9$.

14. Slavutzky SMB, ABBEG C, Gross RFE, Rosa MAC. Mercado de trabalho: perfil do acadêmico de Odontologia da Universidade
Federal do Rio Grande do Sul. Rev Fac Odontol Porto Alegre 2002; 43(2):3-6.

15. Araújo YP, Dimenstein M. Estrutura e organização do trabalho do cirurgião-dentista no PSF de municípios do Rio Grande do Norte. Ciência e Saúde Coletiva 2006; 11(1): 219-227.

16. Brustolin J, Brustolin J, Toassi RFC, Kuhnen M. Perfil do acadêmico de Odontologia da Universidade do Planalto Catarinense-Lages-SC, Brasil. Revista ABENO 2006; 6(1):70-6.

17. Bastos JRM, Aquilante AG, Almeida BS, Lauris JRP, Bijella VT. Análise do perfil profissional de cirurgiões-dentistas graduados na faculdade de odontologia de Bauru - USP entre os anos de 1996 e 2000. J Appl Oral Sci 2003; 11(4): 283-9.

18. Freitas, C.H.S.M. Conflicts in the practice of Dentistry: the autonomy in question. Interface-Comunic. Saúde Educ. 2007; $11(21): 25-38$.

19. Vieira BA. Perfil do estudante de odontologia da Universidade Federal do Ceará e sua visão sobre reforma sanitária e saúde pública. Rio de Janeiro, 1994. 127p. Dissertação (Mestrado) - Faculdade de Odontologia, Universidade Federal Fluminense.

20. Bernabe E, Icaza JL, Delgado-Angulo EK. Reasons for choosing dentistry as a career: a study involving male and female firstyear students in Peru. Eur J Dent Educ 2006; (10):236-241.

21. Aguiar CM, Pessoa MAV, Cmara A C, Perrier R A, Figueireido JAP. Factors involved in the choice of dentistry as an occupation by Pernambuco dental students in Brazil. Journal Dental Education, 2009; 73(12):1401-7.

22. Cruz ACS, Silva EMC. Motivos para a escolha da carreira odontológica. Rev. Assoc. Paul. Cir. Dent. 1996; 10(4): 315-22.

23. Brasil. Ministério da Saúde. Coordenação Nacional de Saúde Bucal. Condições de saúde bucal da população brasileira Projeto SB Brasil 2003 - Resultados Principais. Brasília: Ministério da Saúde, 2004. 68p.

24. Jardim MCAM. Evolução do mercado de trabalho em Odontologia na cidade de João Pessoa - PB. Camaragibe, 1999. 84p. Tese (Doutorado) - Faculdade de Odontologia de Pernambuco, Universidade de Pernambuco.

25. Michel-Crosato E, Calvielli ITP, Biazevic MGH, Crosato E. Perfil da força de trabalho representada pelos egressos da FOUSP (1990-1998). Revista de Pós-Graduação 2003; 10(3): 217-223. 